\title{
VALIDATION OF TOPOLOGY OPTIMIZATION FOR COMPONENT DESIGN
}

\author{
M. Chirehdast \\ S. Sankaranarayanan \\ Alpha Simultaneous Engineering \\ Ford Motor Company \\ Dearborn, MI 48124 \\ S. D. Ambo \\ R. P. Johanson \\ The University of Michigan \\ Department of Mechanical Engineering and Applied Mechanics \\ Ann Arbor, MI 48109-2125
}

\begin{abstract}
$\underline{\text { Abstract }}$
The applicability of structural topology ${ }^{*}$ optimization for the design of automotive components is investigated. Topology optimization is a newly emerging technique that provides design engineers with initial concepts for the layout of structures. Subsequent analysis and optimization are required to refine the initial concept into a detailed final form. The design of the inner panel for a trunk lid is chosen as an example to highlight this process. An existing version of the design is used as the baseline design. The topology of the inner panel for a dominant load case is designed using OPTITOP, a topology optimization program developed at the University of Michigan. A finiteelement model of the inner panel is
\end{abstract}

Topology is a term borrowed from modern mathematics and means properties of geometric configurations that are homeomorphisms (one-to-one mappings) between sets. For practical purposes, the term topology is equivalent to the terms design layout and structural configuration. generated. The model is optimized for stress and displacement requirements using software developed at Ford Motor Company. Significant mass reductions can be achieved when mathematically rigorous concepts suggested by topology optimization are used. As a second example, the design of a suspension arm is studied. For this example, however, only a manufacturable solid model is generated based on the concept suggested by OPTITOP. No detailed analysis and optimization is carried out for this design example.

\section{Introduction}

In today's industrial settings, engineers involved in the design process use their experience extensively to design structures. Using their intuition, the engineers arrive at an initial concept. Further modifications are made based on the results of various structural analysis programs. Employing topology optimization can provide engineers with a rigorously derived initial design that will serve as an 'optimal' starting point. The design procedure suggested in this report, and advocated in numerous publications by the research community in the field of structural optimization, adds more rigor to 
a design phase that has typically been dominated by heuristic approaches.

Structural design optimization is traditionally performed by altering the characteristic sizes of the components (sizing optimization) or by changing their boundaries (shape optimization) while preserving the geometric layout (topology) of the structure. The layout is selected $a$ priori based on the experience and intuition of the design engineer. Such a traditional optimization process can offer only limited improvements in the objective function. On the other hand, using topology optimization the designer has greater flexibility to redefine the structural layout to meet the design goals.

We began investigating the viability of topology optimization for design of automotive components in the spring of 1992. The first phase of these investigations was to evaluate existing options for topology optimization. The finding of that investigation was that the homogenization-based method ${ }^{1}$ developed at the University of Michigan was the best currently available option for topology optimization in a product engineering environment. The focus of this article is to show the merits of applying topology optimization (specifically homogenization) to component design. No extensive literature review for topology optimization and its applications is given here. Interested readers should consult Reference 1 - 4 for extensive reviews of the background, theory, and application of topology optimization.

The remainder of this paper is organized as follows. First the conceptual design of the trunk lid inner panel is discussed in Section 2, followed by the detailed optimization of the design in Section 3. The suspension arm design problem is discussed in Section 4. Concluding remarks summarize the findings of this study.

\section{Inner Panel Design for a Trunk Lid}

The design procedure, discussed in the introduction, has been used to design the stiffener of a trunk lid and is described below.

The baseline design chosen here is the one that existed in June, 1992 (note that the production design has been modified since then, and our baseline design is no longer current.) A review of the structural analysis of the baseline design reveals that the most dominant load in terms of the stresses and displacements applied to the trunk lid is a torsional load in the vertical direction. Figure 1 shows the inner panel with the loads and boundary conditions for this load case.

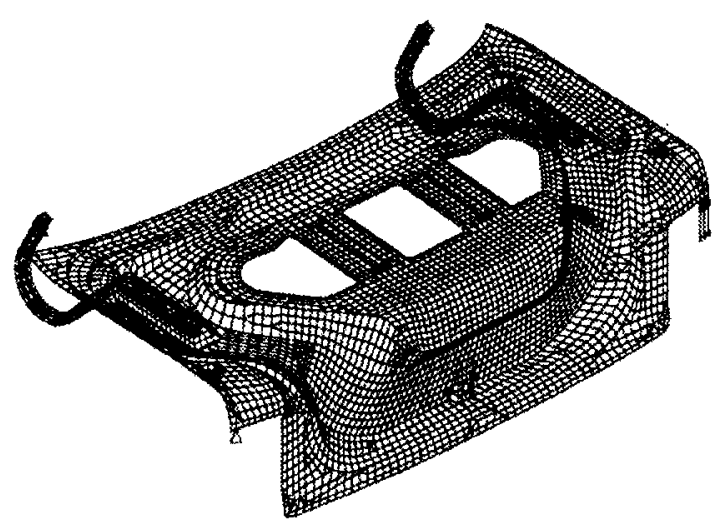

Figure 1. Baseline inner panel for trunk lid - loads and boundary conditions for the dominant load case (torsion.)

\subsection{Topology Design}

The first step in the topology design of the trunk lid is the selection of an appropriate design domain. In this case the outer panel from the baseline design is selected as the initial design domain for topology optimization. The initial design domain is shown in Figure 2. 


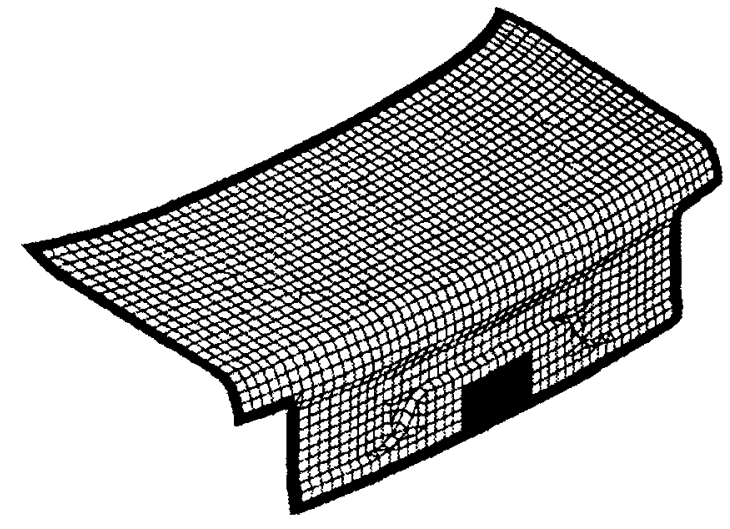

Figure 2. Initial design domain for topology optimization.

The dark areas in the domain are unalterable due to packaging requirements and are considered non-designable for the topology optimization. The torsional loads and the appropriate boundary conditions are applied to this domain.

As discussed earlier, the topology optimization program (OPTITOP) finds the optimal distribution of material for the torsional loads that would represent the stiffest possible inner panel for reinforcing the trunk lid. The input to OPTITOP consists of the finite-element model of the initial design domain with two load cases (to obtain a symmetric design with asymmetric loads and boundary conditions, two load cases are necessary.)

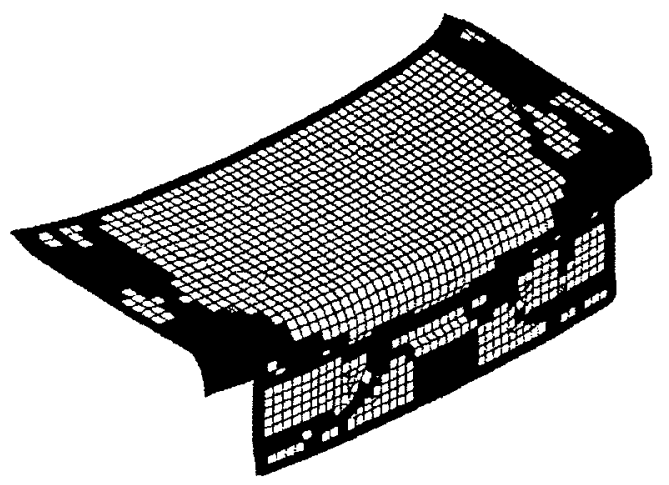

Figure 3. Optimal topology for the inner panel (torsional load case)
The result of topology optimization is shown in Fig. 3. The dark areas in the figure represent the optimal material distribution to maximize the stiffness of the trunk lid. Note that the result is similar to that suggested by intuition in that the topology optimization stiffens the outer boundary of the trunk lid outer panel. This material distribution represents the optimal layout of the inner panel. This image, however, cannot be used as such for further analysis, optimization, or manufacturing. A detailed finite-element model has to be generated from this image.

\subsection{Finite-Element Model Generation and Analysis}

To generate the finite-element model, a design engineer must interpret the image as a structural component. The designer then uses available CAD tools to generate a finite-element model for the inner panel, as shown in Fig. 4. This inner panel is then integrated with the other components of the baseline model replacing the earlier inner panel.

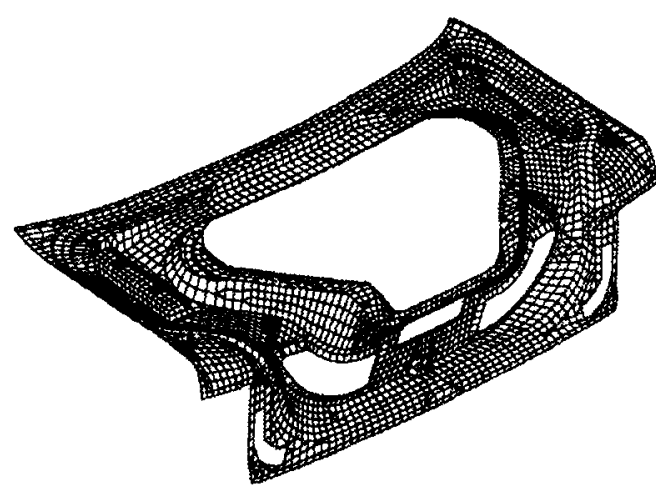

Figure 4. Finite-element model of the inner panel optimum topology

Once the finite-element model is generated, it can be analyzed using a finite-element analysis program, for example, MSC-NASTRAN, to determine the stresses and displacements in the inner panel. At this point conventional structural optimization techniques can be 
applied to finalize the design. Here only sizing optimization is used to determine optimal thicknesses of different panels.

\section{Sizing Optimization of the Trunk Lid}

To meet the stress and displacement requirements similar to the baseline design, two different optimization problems are studied. In the first problem, the objective is to minimize the mass of the trunk lid where the thicknesses of the inner, outer, and inner attachment reinforcement panels are used as design variables for the optimization. The displacements at the load application points were constrained to be less than a specified amount per the baseline design. The maximum von Mises stress in all the panels in the trunk lid excluding the hinge assembly arm are constrained to be less than the yield stress of the material. The thicknesses used as design variables are required to be within certain bounds as prescribed by manufacturing and other requirements. This optimization problem can be mathematically represented as follows:

Minimize $m\left(t_{I}, t_{O}, t_{R}\right)$

mass as a function of $t_{I}, t_{O}$, and $t_{R}$ respectively, the thicknesses for the inner, outer, and reinforcement panels,

\section{Subject to}

$\mathrm{u} \quad \leq \quad \mathrm{u}_{\max }$
maximum displacement

$\sigma \leq \sigma_{\max }$

stress constraints for panels

$\mathrm{t}_{\mathrm{I} \min } \leq \mathrm{t}_{\mathrm{I}} \leq \mathrm{t}_{\mathrm{I} \max }$

$\mathrm{t}_{\mathrm{O} \text { min }} \leq \mathrm{t}_{\mathrm{O}} \leq \mathrm{t}_{\mathrm{O} \max }$

$t_{R \text { min }} \leq t_{R} \leq t_{R \text { max }}$.

manufacturing bounds on panel

thicknesses
The second optimization problem is similar to the first problem, but here the outer panel thickness is equal to the one in the baseline design. The stress and displacement constraints remain the same as in the first problem.

The sizing optimization has been carried out using a software based on CONMIN that employs a feasible direction method to solve nonlinear programming problems ${ }^{5}$. The results of the detailed design optimization are summarized in Table 1, and indicate that significant mass reductions were realized.

Table 1: Percentage improvement versus baseline design

(reduction relative to baseline design)

\begin{tabular}{|l|c|c|}
\hline $\begin{array}{l}\text { Performance } \\
\text { Measure }\end{array}$ & Design 1 & Design 2 \\
\hline Mass of Inner Panel & $32 \%$ & $37 \%$ \\
\hline Mass of Trunk Lid & $17 \%$ & $12 \%$ \\
\hline $\begin{array}{l}\text { Maximum Stress } \\
\text { (Inner Panel) }\end{array}$ & $24 \%$ & $21 \%$ \\
\hline Max. Displacement & $24 \%$ & $23 \%$ \\
\hline
\end{tabular}

The trunk lid models with the inner panel designed by topology optimization were then analyzed for additional load cases that were analyzed for the baseline design. The design that resulted from the optimization procedure offered satisfactory structural performance in all load cases.

\section{Suspension Arm Design}

Figure 5 shows a two-dimensional representation of the loading and boundary conditions applied to the design of a lower control arm for a vehicle suspension. Note that the primary loading condition is a vertical load applied at point $B$, and the design is constrained to pivot about the 1-2 axis. The boundary conditions and relative loads are shown in Table 2 . Figure 6 shows a three-dimensional model of the initial design domain and applied boundary and loading conditions for the design of a suspension arm. The upper 
curve represents the packaging constraint for the wheel, and the spring bucket must be included to secure the functionality of the spring. This model contains approximately 12,000 elements and serves as the input to the topology optimization program OPTITOP.

Figure 7 shows the output of OPTITOP for a given amount of material that represents $20 \%$ of the material of the original design domain, shown in Figure 6; different colors represent different densities at the element level. The material allocation provides an approximation (due to discretization) to the stiffest possible design for this specified amount of material.

Figure 8 shows a manufacturable design that has been obtained based on the optimal material distribution shown in Figure 7. This transition has been made semi-automatically using a program that generates smooth surfaces between given sections. No final structural analysis has been performed for this design. Therefore, no comparison can be made between the baseline design and the one illustrated in Figure 8 in terms of their weights.

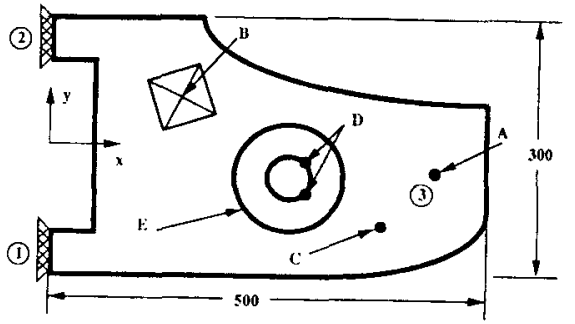

Figure 5: Loading and boundary conditions for suspension arm design.

Table 2: Relative magnitiudes of applied loads for suspension arm design.

\begin{tabular}{|c|c|c|c||c|c|}
\hline $\begin{array}{c}\text { Loca } \\
\text {-tion }\end{array}$ & $\begin{array}{c}\text { Magn. } \\
(\mathrm{X})\end{array}$ & $\begin{array}{c}\text { Magn. } \\
(\mathrm{Y})\end{array}$ & $\begin{array}{c}\text { Magn. } \\
(\mathrm{Z})\end{array}$ & $\begin{array}{c}\text { Bound. } \\
\text { Cond. }\end{array}$ & \\
\hline A1 & -0.1152 & 0.4651 & 0 & 1 & 12346 \\
\hline A2 & -0.1028 & 0.1881 & 0 & 2 & 12346 \\
\hline B & -0.1978 & 0.1816 & 1 & 3 & 3 \\
\hline C & 0 & 0 & -0.1085 & & \\
\hline
\end{tabular}

\begin{tabular}{|c|l|l|l||l|l|}
\hline $\mathrm{D}$ & 0 & 0 & -0.1085 & & \\
\hline $\mathrm{E}$ & 0 & 0 & 0.6562 & & \\
\hline
\end{tabular}

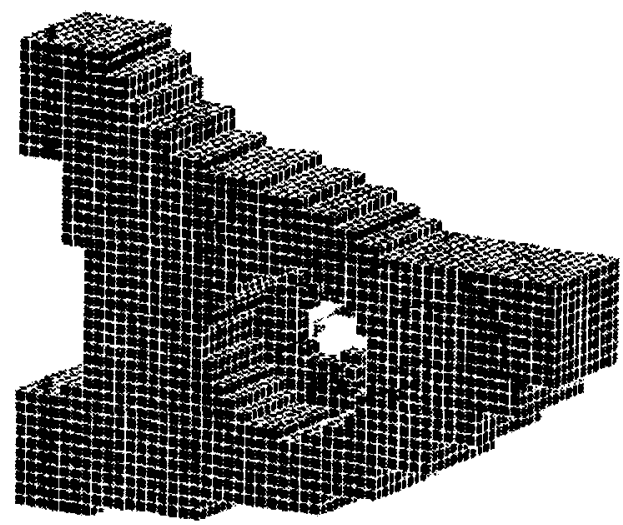

Figure 6. Three-dimensional input model to OPTITOP for the suspension arm design .

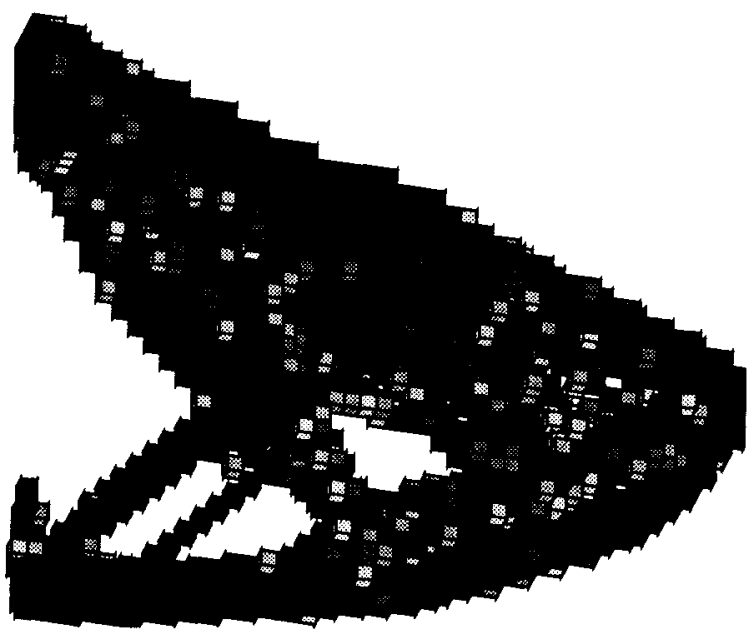

Figure 7. Output of OPTITOP for the suspension arm design (volume constraint is $20 \%$ ). 


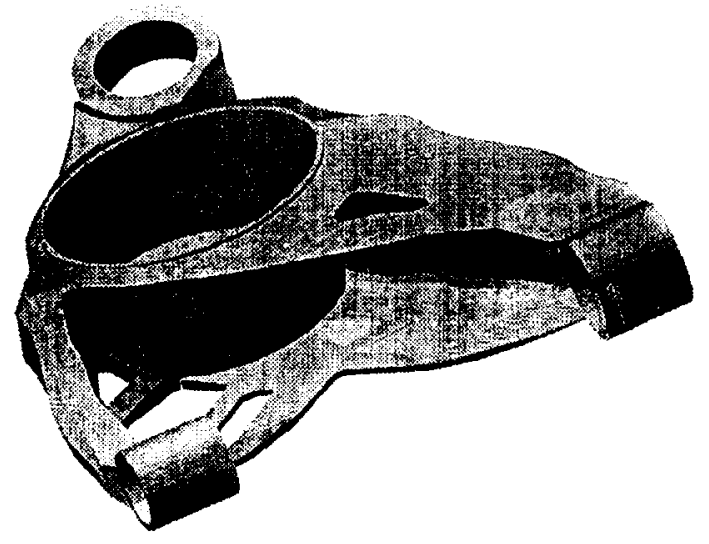

Figure 8. A manufacturable representation of the design shown in Figure 7.

\section{Concluding Remarks}

Topology optimization is introduced as a viable tool for structural design, where an initial optimized concept can be obtained. This concept requires further refinements in subsequent analysis and optimization. The merits of using topology optimization to arrive at a rigorous initial concept are shown using the example of a trunk lid. The inner panel that is designed using topology optimization weighs less and is stiffer than the baseline design. Topology optimization can by no means replace the experience and judgment of engineers in the complex process of designing structural components. However, this technique should be viewed as a useful tool to effectively guide their efforts in the initial stages of design.

As the engineering community gains more experience with rigorous methods of structural analysis, design, and optimization, further improvements to programs (such as those discussed here) become obvious and necessary.

\section{Acknowledgments}

The project presented here is a collaborative team effort involving many individuals from Ford and the University of Michigan. We would like to thank B. Barthelemy, C. Chuang, R. I. Devries, D.
Phan, S. Sharma, Y. Song, and R. J. Yang of Ford Motor Company and N. Kikuchi, P. Y. Papalambros, and Y. K. Park of the University of Michigan.

\section{References}

1 Suzuki, K., and Kikuchi, N., 1991, Homogenization Method for Shape and Topology Optimization. Computer Methods in Applied Mechanics and Engineering, Vol. 93, pp. 291-318.

2 Bendsoe, M.P and Kikuchi, N., 1988, Generating Optimal Topologies in Structural Design Using a Homogenization Method, Comp. Meth. Appl. Mech. Eng., Vol. 71,pp.197-224.

3 Bremicker, B., Chirehdast, M., Kikuchi, N., Papalambros, P., 1991, Integrated Topology and Shape Optimization in Structural Design. Mechanics of Structures and Machines, Vol. 19, No. 4, pp. 551-587.

4 Chirehdast, M., Gea, H. C., Kikuchi, N., Papalambros, P., 1994, Structural Configuration Examples of an Integrated Design Process, to appear in the $A S M E$ Journal of Mechanical Design.

5 Vanderplaats, G. N., 1973, CONMIN - A Fortran Program for Constrained Function Minimization, User's Manual, NASA TM X-62, 282. 